States of Unesco. The coupons may be used to buy books on education, science and culture, the importation of which is prohibited by importing laws. They can also be used for subscriptions to periodicals, for the purchase of individual volumes or issues and for the payment of photo copies. The coupons will be available in denominations ranging from 25 cents to 10 dollars. Other countries where the coupons will be on sale are : China, Czechoslovakia, France, India and Poland. In addition, a limited free distribution, financed by Unesco, will be made in Austria, China, Czechoslovakia, Greece, Hungary, Italy, the Philippines and Poland. The coupons of each participating country will be handled by its own national distributing body, and in Great Britain this function is being undertaken by Book Tokens, Ltd., 28/30 Little Russell Street, London, W.C.1, from whom all particulars may be obtained. It is pointed out that as the amount of dollars available is limited, coupons will be allocated on a priority basis, and the needs of educational, scientific and cultural institutions serving a large-public will be given first consideration.

\section{Northerh Advisory Council for Further Education}

IN March and April of 1947, the Minister of Education issued direetions to local education authorities to prepare and subinit schemes for further education and plans for county colleges. In Circular 133, conteinge directions for further education, the Mífister reminded local education authorities that they are required when preparing their schemes to "have regard to facilities provided by other bodies and to consult such bodies and local education authorities for adjacent areas". The procedure for the consultations referred to in Circular 133 was discussed by the Northern Advisory Council for Further Education at its meeting in October 1947, when it was decided that the first step should be the preparation of an agreed draft by a committee consisting of the 'chief education officers' of the constituent authorities. Its report has now been prepared and was approved by the Council in November 1948. Adult education, county colleges, community centres and other kinds of further education were considered to lie outside the scope of the Committee, and its deliberations were confined to technical, com. mercial and art education as provided in the main colleges. Starting from the broad principle that each authority should be free to provide for its own residents up to the national certificate stage, provided that numbers justified this measure, the Committee has worked out a detailed programme in which technical education in the north-east area could function as an integrated regional scheme. Implementation of the recommendations in this report would do much to provide Great Britain with adequate numbers of the technically trained persons who are so urgently required. Copies of the report may be obtained from 43 Eldon Place, Barras Bridger.Neweastle-upon-Tyne, 1.

\section{Magnetic Field of the Sun}

A RECENT paper by Prof. S. Chapman (Mon. Not. Roy. Astro Soc., MaNo. 3; 1948), with the title "Solar Magnetisn fapy Athe Suggested Fundamental Magnetization Py Rotation", deals with the difficulties in acepfing the 'fundamental' hypothesis, recently repised by Blackett. According to this hypothesis, the general magnetic fields of the earth, the sun and of certain stars introduce a new fundamental property of rotating bodies which can be expressed by the formula $P / W=\beta G^{1 / 2} / 2 c, P$ being the axial component of the dipole magnetic moment, $W$ the angular momentum, $G$ the constant of gravitation, $c$ the speed of light, and $\beta$ a factor of the order unity (for the earth it is 0.3 ). It was pointed out by Bullard that the fundamental hypothesis seems to imply a downward decrease of intensity of the geomagnetic field within the earth, and measurements since made in South Africa and in England provisionally support the hypothesis (see Nature, 160, 746; 1947; 161, $462 ; 1948)$. Chapman develops the consequences of the hypothesis-in particular as to the nature of the internal fields of the earth and sun-basing his calculation on the density distribution given by Blanch, Lowan, Marshak and Bethe for the point convective model of the sun, and taking into account a non-uniform distribution of the angular velocity approximating to that given by Schwarzschild. The corresponding moment of inertia and angular momentum of the sun are calculated, and $\beta$ is estimated to be 2 if the sun's polar magnetic intensity is 50 gauss; but accepting Thiessen's recent value of 10 gauss, or less, $\beta$ cannot exceed $0 \cdot 4$, which is close to $0 \cdot 3$, the value of $\beta$ for the earth. The inferred intensity at the centre is a few thousand gauss, similar to that of sunspot magnetic fields. The fundamental theory seems incapable of explaining the existence of the intense sunspot magnetic field, and this weakens the case for postulating a new fundamental property of matter to explain the general magnetic field. It is admitted, however, that it is remarkable, and may be profoundly significant, that the ratios of the values of $P$, extending over a considerable range, for the earth, the sun, and certain stars, to the associated values of $W$, differ by less than one factor of ten.

\section{Suggestions for Science Teachers in Devastated Countries}

UNEsco haf recently published an illustrated booklet shyting how teachers lacking elementary scientific oquipment can make apparatus from simple everyday materials. The booklet has been prepared by Mr. J. P. Stephenson, science master at the City of London School, who first explains how science teaching can be commenced without the use of apparatus and then shows how equipment for experiments in astronomy, meteorology, heat, light, mag. netism, electricity, chemistry and biology can be improvised from materials such as wood, glass-tube, wire, nails, bottles and other household articles.

A note is also included on the use of visual aids in science teaching together with a description of recent laboratory materials, such as plastics and alloys, and a section on laboratory receipts, charts and logarithm tables. The booklet is being distributed free by Unesco to schools in Greece, Poland, Czecho. slovakia, Austria, Hungary, Italy, China and the Philippines, with the hope that it may lead to further improvisation in apparatus for elementary science teaching. It should be interesting later to see what influence this booklet has on the teaching of science in the recipient countries.

\section{Paper Supplieg for Books}

IN a writen reply to a question in the House of Commgns, the President of the Board of Trade states that/rom March 6 the licensing arrangements for paper will be considerably simplified. As a result, paper for books, stationery, boxes, cartons and many minor uses should be freely available without in. 